\title{
Labyrinthe
}

15 | 2003

Territoires : questions d'images

\section{Fredrik Barth, Balinese Worlds, Chicago/Londres, University of Chicago Press, 1993}

\section{Marc Aymes}

\section{(2) OpenEdition \\ 1 Journals}

\section{Édition électronique}

URL : http://journals.openedition.org/labyrinthe/483

DOI : $10.4000 /$ labyrinthe.483

ISSN : 1950-6031

Éditeur

Hermann

\section{Édition imprimée}

Date de publication : 1 juin 2003

\section{Référence électronique}

Marc Aymes, «Fredrik Barth, Balinese Worlds, Chicago/Londres, University of Chicago Press, 1993 »

Labyrinthe [En ligne], 15 | 2003, mis en ligne le 24 juillet 2008, consulté le 02 mai 2019. URL : http:// journals.openedition.org/labyrinthe/483 ; DOI : 10.4000/labyrinthe.483

Ce document a été généré automatiquement le 2 mai 2019.

Propriété intellectuelle 


\title{
Fredrik Barth, Balinese Worlds, Chicago/Londres, University of Chicago Press, 1993
}

\author{
Marc Aymes
}

1 Le champ des sciences humaines et sociales françaises bruisse de références bibliographiques aux ouvrages de l'anthropologue norvégien Fredrik Barth: Models of Social Organization (1966), Ethnic Groups and Boundaries (collectif d'articles, 1969), Process and Form in Social Life (1981). Pourtant, il n'est pas si fréquent (un rapide survol internautique du formidable catalogue Sudoc en atteste) que ces titres honorent les fonds des bibliothèques universitaires nationales, et seul Ethnic Groups a fait l'objet de traductions ${ }^{1}$. En quelques publications, une fine équipe de chercheurs, soucieuse d'engager les Annales dans un " tournant critique », a témoigné de ce que l'anthropologue norvégien lui avait apporté d'énergie cinétique - tout en confessant volontiers que l'accélération décisive avait été transmise par le relais de la microstoria italienne ${ }^{2}$. Mais Balinese Worlds, paru en 1993, ne semble guère à ce jour avoir retenu l'attention des chercheurs français. Pourtant, Barth y propose de nouvelles et stimulantes pièces pour le puzzle que les sciences sociales s'emploient à reconstituer. De quoi redoubler d'imagination et de patience pour interroger la variabilité du monde social, ainsi que la géométrie instable des comportements et des pratiques. De quoi aussi justifier d'en proposer, dix ans après, un compte rendu anniversaire ${ }^{3}$.

2 Commençons par un rappel: les travaux de Barth participent de ce qu'il est convenu d'appeler l'« anthropologie sociale». C'est-à-dire que, à l'opposé des approches dites " culturalistes », ils privilégient le caractère relationnel des réalités humaines observées. L'interaction entre individus occupe, en d'autres termes, une place essentielle dans le dispositif de recherche : à la fois unité d'observation et principe de compréhension de l'anthropologue. Les pratiques et les processus qui se jouent dans les relations sociales, soutient Barth, troublent sans cesse la surface trop lisse des notions tenues pour acquises par l'anthropologie culturaliste : une «culture » tient principalement dans les pratiques 
qui la mettent à l'épreuve, en renouvellent la pertinence ou l'invalident ; une "identité " ne vaut qu'en vertu des processus de son attribution, de sa perpétuation ou de son renversement. Tel est, brièvement, le portrait-robot que l'on peut proposer de l'anthropologie sociale en général, ou de celle de Barth en particulier.

Cela étant dit, Balinese Worlds présente, en regard des ouvrages antérieurs de son auteur, des spécificités significatives. Adoptons une perspective cavalière : soit Ethnic groups notre ouvrage de référence ${ }^{4}$, enjambons les vingt années de travaux qui le séparent de Balinese Worlds; qu'observe-t-on ? La notion d'» identité » charpentait le premier ouvrage, elle a disparu corps et biens dans le second. Celle de «frontière » (boundary), à laquelle Barth avait pourtant donné un rôle heuristique crucial dans le développement de sa démarche, est explicitement - quoique fugitivement - récusée (p. 4). Cependant, plusieurs marques de fabrique demeurent: d'une part, l'approche par la pratique des acteurs, puisque le primat est donné au "people's "here" and "now", centering on themselves» (p.4); d'autre part, le souci de produire un modèle à caractère génératif, processuel. S'y ajoute simplement une interrogation sur la notion de "civilisation complexe ", qui sert de relais au problème de la multiplicité et de l'irréductibilité des données auxquelles le monde social confronte l'anthropologue. Et ce questionnement donne lieu à un travail sur la notion de " culture ", avec laquelle Barth semblait en délicatesse vingt ans plus tôt.

Réflexion sur la complexité et la prolifération des activités humaines en société, Balinese Worlds est un mastodonte, qui - les contraintes éditoriales sont-elles passées par là ? semble un peu à l'étroit dans ses trois cent cinquante pages. Mastodonte, mais bondissant: dense, le propos avance par rebonds, par retours incessants sur les conclusions précédemment tirées pour en (ré) estimer la portée. L'ouvrage ne cherche nullement à masquer ses réglages, ses changements de point de vue : Barth le qualifie de "slow and cumulative account»(p.8). Le corps de l'étude est rythmé par quatre ensembles de chapitres (les "parties » numérotées 2 à 5), selon une scansion qui fait alterner une succession de textes de portée, disons, monographique ou thématique, d'une part, et d'autre part des essais de synthèse. Ces derniers (chapitres 6, 10 et, dans une moindre mesure, 15) apparaissent, à la lecture, comme de véritables chevilles ouvrières de l'ouvrage : ils sont en effet le lieu où Barth effectue la remise à plat des problèmes posés et cherche à mettre en place ce que les chapitres immédiatement précédents ont apporté à leur résolution... pour mieux pouvoir proposer alors un déplacement de leurs perspectives. Encore dans la conclusion de l'ouvrage, le voilà affairé à débrouiller une « énigme " - en l'occurrence, le fait que des individus adoptent des comportements observables similaires malgré des « constructions culturelles de la réalité » divergentes. La démarche de Barth apparaît clairement ici : pousser à bout les interrogations sur les problèmes, c'est-à-dire sur la manière dont ils sont posés (et non pas "se posent "), décortiquer leurs présupposés et leurs conditions de pertinence (p.341-342). Leaving no stone unturned.

Comment «rendre compte " d'une telle hydre? Une tête après l'autre, je vous prie : à titre de points de repère, rappelons les grandes lignes de chaque partie. Dans la partie 2, l'anthropologue s'attache à planter le décor social ou, comme il le dit, à «définir et structurer les arènes dans le cadre desquelles les gens agissent » (p. 104, c'est l'auteur qui souligne). Voilà qui nous rappelle le Barth des années 1960: dans Ethnic Groups, déjà, l'article qu'il consacrait à « l'identité pathane » faisait une large place aux « forums [...] qui permettent de mener à bien et d'exhiber un comportement pertinent ${ }^{5} »$. Par contraste, la partie 3 porte sur «comment les gens agissent», ce qui renvoie aux 
«significations éprouvées » (experienced meanings) des actions, aux "préoccupations » ( concerns) des individus (p. 104). Disons que se dessine ici une sorte de déconstruction (en écartant le sens philosophique de ce terme, faute d'avoir mené la réflexion qui pourrait en révéler la pertinence) : le caractère systématique, «formel» (p.157) des organisations ou institutions sociales est dissout en un éparpillement de concerns. Dans le mouvement de pensée ainsi défini, la partie 4 peut apparaître comme la recherche de nouveaux éléments structurants, qui cette fois préserveraient (p. 268) la diversité observée (là où les formes institutionnelles uniformisaient le varié): c'est ce que Barth appelle les "traditions de connaissance " (traditions of knowledge), définies en tant que courants de polarisation des préoccupations, et considérées comme des " paradigmes pour l'action et l'interprétation des événements » (p. 267) : à savoir, l'islam, l'hindouisme, les hiérarchies de l'autorité politique, l'occidentalisation, la sorcellerie. Il précise: "Chacune est un conglomérat historique de matériaux, soudés par l'action d'une organisation sociale effective [functioning] et non par la cohérence logique de ses idées constitutives » (p. 266). Et encore: "Chacune de ces traditions différentes, en tant que paradigme, n'a pas de cohérence interne et n'est pas transparente à elle-même ; elles ne s'excluent pas non plus mutuellement, au sens où une personne ne pourrait en embrasser plusieurs» (p. 267). Mais se pose tout de même le problème de la cohabitation de ces traditions of knowledge, c'est-à-dire en pratique des conditions dans lesquelles des personnes se référant à des traditions différentes peuvent entrer en «interaction signifiante» (p. 267). La partie 5 délivre à ce sujet une volée de questions-réponses, où le caractère expérimental et pragmatique de la démarche de Barth se découvre, effectivement : on s'y interroge sur la « diversité des expériences et des jugements à partir d'événements identiques » (p. 286), et sur les conditions d'une " convergence dans les significations construites par les gens » (p. 342); on y établit qu'une tradition n'est "pas maintenue à cause de [sa] force épistémologique [...], mais en raison de la force démontrée de sa pratique sociale » (p. 314) ; et puis il y a, à côté des traditions de connaissance, «l'expressivité de symboles clés » (p. 324), des « imageries » caractérisées par la polyvalence et le flux sémantiques, une « productivité poétique » (p. 336) qui traverse la société de part en part (p. 329).

6 Au final, on le comprend, c'est d'une tentative pour élaborer une anthropologie du savoir qu'il s'agit. Le savoir des Balinais n'est bien sûr pas seul en cause, tant Barth se plaît à faire oublier la frontière du " eux » au "nous ». De fait l'interrogation porte tout autant sur les savoirs possibles de l'anthropologue, au premier rang desquels figure un savoirvivre $^{6}$ : délaissant « les entretiens formels et la théorie abstraite » (p. 161), Barth préfère en effet voir sa vie «envahie» par les préoccupations balinaises (p. 346), car ainsi se perçoit « la différence entre construire une tradition de connaissance et vivre une vie » (p. 322). D'aucuns estimeront que c'est pousser trop loin la démarche compréhensive, et assurément les enjeux sont considérables. Mais épistémologie, philosophie que tout cela. "Sage sans idée ${ }^{7}$ ", Fredrik Barth préfère nous offrir en partage la perplexité du vivant et la poésie de l'humain. En toute disponibilité. 


\section{NOTES}

1. L'introduction du recueil d'une part: «Les groupes ethniques et leurs frontières » [traduction par P. Poutignat et J. Streiff-Fenart], dans J. Bardolph, P. Poutignat, J. Streiff-Fenart (dir.), Théories de l'ethnicité, Paris, Puf, p. 203-249 ; l'article monographique signé F. Barth d'autre part: «L'identité pathane et sa préservation» [traduction (partielle) par M. Aymes et S. Péquignot], Labyrinthe, $n^{\circ}$ 7, p. 43-65.

2. Voir ainsi Maurizio Gribaudi, Itinéraires ouvriers. Espaces et groupes sociaux à Turin au début du XXe siècle, Paris, Éditions de l'EHESS 1987, p. 24-25 notamment; et PaulAndré Rosental, «Construire le « macro » par le « micro » : Fredrik Barth et la microstoria », dans Revel, Jacques (dir.), Jeux d'échelles. La micro-analyse à l'expérience, Paris, Gallimard-Le Seuil, coll. « Hautes Études », 1996, p. 141-159.

3. En France, d'après le catalogue du Sudoc, Balinese Worlds est disponible dans les bibliothèques suivantes : universités Lille I et Paris VIII, Inalco, musée de l'Homme. Il est aisé de se procurer l'ouvrage sur les principaux sites Internet de vente en ligne, mais attention, son prix (normalement situé autour de 25-30\$) peut varier du simple au triple.

4. Raisons invocables pour un tel choix : les traductions mentionnées plus haut, et les rééditions multiples (en 1994 et 1998).

5. «L'identité pathane... », art. cit., p. 51.

6. Bio-logie, donc. Rappelons que Barth s'est consacré à celle-ci avant de s'orienter vers l'anthropologie.

7. Mots librement empruntés à François Jullien: Un sage est sans idée, Paris, Seuil, coll. « L'ordre

philosophique », 1998. 